\title{
National Recommendations for Preconception Care: The Essential Role of the Family Physician
}

\author{
Anne L. Dunlop, MD, MPH, Brian Jack, MD, and Keith Frey, MD, MBA
}

The Centers for Disease Control and Prevention have published national recommendations for improving preconception health and health care in response to unfavorable aspects of the health status of women and children in the United States. The publication explains that the national recommendations are part of a strategic plan for improving preconception health through the provision of clinical care as well as the promotion of changes in individual behaviors, health policy, and public health strategies. The concept of preconception care has been articulated for well over a decade but has not become part of the routine practice of family medicine. Because all women of reproductive age presenting to the primary care setting are candidates for preconception care, the essential and critical role of family physicians in the provision of preconception care is apparent. As a specialty, we are now challenged to devise ways to effectively translate the concept of preconception care into clinical reality. (J Am Board Fam Med 2007;20:81-4.)

The Centers for Disease Control and Prevention, together with a Select Panel of external partners, recently published national recommendations for improving preconception health and health care. ${ }^{1}$ The intent of the recommendations is to address unfavorable aspects of the health status of women and children in the United States, including rates of adverse birth outcomes (preterm and low birth weight deliveries, infant deaths, and birth defects), maternal pregnancy complications, and unintended pregnancies - all which are higher than goals outlined in Healthy People 2010. ${ }^{2}$

The national recommendations should be viewed as a strategic plan for improving preconception health through the provision of clinical care as well as the promotion of changes in individual behaviors, health policy, and public health strategies. The national recommendations with background

This article was externally peer reviewed.

Submitted 19 August 2006; revised 24 October 2006; accepted 30 October 2006.

From Emory University School of Medicine, Atlanta, GA (ALD); Boston Medical Center, Boston, MA (BJ); and Mayo Clinic Scottsdale, Scottsdale, AZ (KF).

Conflict of interest: none declared.

Corresponding author: Anne L. Dunlop, MD, MPH, Department of Family and Preventive Medicine, Emory University School of Medicine, 735 Gatewood Road NE, Atlanta, GA 30322 (E-mail: amlang@emory.edu). information, review of the existing evidence, and references for incorporating preconception care into practice are found on CDC web sites. ${ }^{3,4} \mathrm{~A}$ brief description of the 10 key recommendations are found in Table 1.

The concept of preconception care has been articulated for well over a decade, ${ }^{5-20}$ yet has not become part of the routine practice of family medicine. Lack of physician knowledge of recommended interventions is one barrier to the provision of preconception care. The CDC publication addresses the knowledge barrier by outlining 14 specific preconception health care interventions for which clinical practice guidelines and evidence of effectiveness exist (Table 2). A complete overview of existing clinical practice guidelines that address preconception care and the evidence supporting the recommended interventions is available. ${ }^{21}$

Other recognized barriers to the provision of preconception care include lack of patient knowledge of and demand for services, lack of physician time, and lack of insurance coverage. Six of the 10 key recommendations address these barriers through public health outreach and social marketing programs (recommendations 1, 2, 8), policy strategies (recommendation 7), and health services research initiatives (recommendations 9, 10).

The essential and critical role of family physicians in the provision of preconception care is ap- 
1 Individual responsibility across the life span. Encourage each woman and every couple to have a reproductive life plan.

2 Consumer awareness. Increase public awareness of the importance of preconception health behaviors and increase individuals' use of preconception care services using information and tools appropriate across varying age, literacy, health literacy, and cultural/linguistic contexts.

3 Preventive visits. As a part of primary care visits, provide risk assessment and counseling (education and health promotion) to all women of childbearing age to reduce risks related to the outcomes of pregnancy.

4 Interventions for identified risks. Increase the proportion of women who receive interventions as follow up to preconception risk screening, focusing on high priority interventions (ie, those with high population impact and sufficient evidence of effectiveness).

5 Interconception care. Use the interconception period to provide intensive interventions to women who have had a prior pregnancy ending in adverse outcome (eg, infant death, low birthweight, or preterm birth).

6 Prepregnancy check ups. Offer, as a component of maternity care, one pre-pregnancy visit for couples planning pregnancy.

7 Health coverage for low-income women. Increase Medicaid coverage among low-income women to improve access to preventive women's health, preconception, and interconception care.

8 Public health programs and strategies. Infuse and integrate components of preconception health into existing local public health and related programs, including emphasis on those with prior adverse outcomes.

9 Research. Augment research knowledge related to preconception health.

10 Monitoring improvements. Maximize public health surveillance and related research mechanisms to monitor preconception health.

Table 2. Preconception Interventions with Evidence for Improving Pregnancy Outcomes

Intervention Proven Health Effect

Folic acid supplementation

Rubella vaccination

Diabetes management

Hypothyroidism management

Hepatitis B vaccination for at risk women

HIV/AIDS screening and treatment

STD screening and treatment

Maternal PKU management

Oral anticoagulant use management

Antiepileptic drug use management

Accutane use management

Smoking cessation counseling

Eliminating alcohol use

Obesity control
Reduces occurrence of neural tube defects by two thirds.

Provides protection against congenital rubella syndrome.

Substantially reduces the 3 -fold increase in birth defects among infants of diabetic women.

Adjusting levothyroxine dosage early in pregnancy protects proper neurologic development.

Prevents transmission of infection to the infant and eliminates the risk to the woman of hepatic failure, liver carcinoma, cirrhosis, and death due to HBV infection.

Allows for timely treatment and provides women (or couples) with additional information that can influence the timing of pregnancy and treatment.

Reduces the risk of ectopic pregnancy, infertility, and chronic pelvic pain associated with Chlamydia trachomatis and Neisseria gonorrboea and reduces the possible risk to the fetus of fetal death and physical and developmental disabilities, including mental retardation and blindness.

Prevents babies from being born with PKU-related mental retardation.

Switching women off teratogenic anticoagulants (ie, warfarin) before pregnancy avoids harmful exposure.

Changing to a less teratogenic treatment regimen reduced harmful exposure.

Preventing pregnancy for women who use isotretinoin (Accutane) or ceasing isotretinoin use before conception, eliminates harmful exposure.

Completing smoking cessation before pregnancy care can prevent smokingassociated preterm birth, low birth weight, or other adverse perinatal outcomes.

Controlling alcohol binge drinking and/or frequent drinking before pregnancy prevents fetal alcohol syndrome and other alcohol-related birth defects.

Reaching a healthy weight before pregnancy reduces the risks of neural tube defects, preterm delivery, diabetes, cesarean section, and hypertensive and thromboembolic disease that are associated with obesity.

PKU, phenylketonuria. 
parent. Family physicians provide much of the health maintenance, family planning, and chronic disease care for women, and strive to maintain continuity of care (often before, after, and between pregnancies) and close relationships with their patients. A recent survey documents that women prefer to receive preconception health information from their primary care physicians. ${ }^{22}$ As a specialty, we are now challenged to effectively translate the concept of preconception care into clinical reality. This is a clinical and public health domain where Family Medicine must assume a leadership role or be usurped by other disciplines with far less impact than could otherwise be realized.

In the short term, family physicians could do 2 things to promote preconception health and health care. First, ask every women of reproductive age whether she intends to become pregnant in the next year. Asking every woman about her reproductive intentions promotes the idea that pregnancies should be intended and planned by providing contraception to the woman who does not intend to conceive and promotes the initiation of preconception care strategies for women if and when they do desire to become pregnant. Second, inform women that health conditions and medications can affect pregnancy outcomes and that pregnancy can affect a woman's health.

In the longer term, aspects of the national recommendations could be incorporated into the Future of Family Medicine Project's "New Model" of family medicine, which promotes the provision of team-based, patient-centered care and a commitment to providing an essential "basket of services." ${ }^{23} \mathrm{We}$ advocate that the intentional provision of preconception care be included in family medicine's essential "basket of services."

All women of reproductive age and potential presenting to the primary care setting are considered candidates for preconception care. ${ }^{1}$ Some have expressed concern that the national recommendations excessively focus the health care encounter on a woman's reproductive potential. However, the intent of the national recommendations is to empower women by promoting reproductive planning linked with the provision of contraception to prevent unintended pregnancies and by providing them with relevant information to make their own informed decisions.

\section{References}

1. Johnson K, Posner SF, Biermann J, et al; CDC/ ATSDR Preconception Care Work Group; Select Panel on Preconception Care. Recommendations to improve preconception health and health careUnited States. A report of the CDC/ATSDR Preconception Care Work Group and the Select Panel on Preconception Care. MMWR Recomm Rep 2006;55(RR-6):1-23.

2. US Department of Health and Human Services. Healthy People 2010. 2nd ed. With Understanding and Improving Health and Objectives for Improving Health. 2 vols. Washington DC: US Government Printing Office; 2000.

3. Centers for Disease Control and Prevention [homepage on the Internet]. Atlanta: Centers for Disease Control and Prevention; c2006 [cited 2006 Oct 30]. Morbidity and Mortality Weekly Report (MMWR). Available from http://www.cdc.gov/ mmwr/.

4. Centers for Disease Control and Prevention [homepage on the Internet]. Atlanta: Centers for Disease Control and Prevention; c2006 [cited 2006 Oct 30]. Preconception care. Available from http:// www.cdc.gov/ncbddd/preconception/default.htm.

5. Committee to Study the Prevention of Low Birthweight, Division of Health Promotion and Disease Prevention, Institute of Medicine. Preventing low birth weight. Washington DC: National Academy Press; 1985.

6. Moos MK, Cefalo RC. Preconceptual health promotion: a focus for obstetric care. Am J Perinatol 1987; 47:63-7.

7. Caring for our future, the content of prenatal care: a report of the Public Health Service Expert Panel on the Content of Prenatal Care. Washington DC: Public Health Service, Department of Health and Human Services; 1989.

8. Toward improving the outcome of pregnancy (TIOP II): the 90s and beyond. White Plains (NY): March of Dimes Birth Defects Foundation; 1993.

9. Jack BW, Culpepper L. Preconception care: risk reduction and health promotion in preparation for pregnancy. JAMA 1990;264:1147-9.

10. Jack BW, Culpepper L. Preconception care. J Fam Pract 1991;32:306-15.

11. Allaire AD, Cefalo RC. Preconceptional health care model. Eur J Obstet Gynecol Reprod Biol 1998;78: 163-8.

12. Reynolds HD. Preconception care: an integral part of primary care for women. J Nurse Midwifery 1998; $43: 445-8$.

13. Bernstein PS, Sanghvi T, Merkatz IR. Improving preconception care. J Reprod Med 2000;45:546-52.

14. Hobbins D. Prepping for healthy moms \& babies. Making the case for preconception care \& counseling. AWHONN Lifelines 2001;5:49-54. 
15. Frey KA. Preconception care by the non-obstetrical provider. Mayo Clin Proc 2002;77:469-73.

16. Moos MK. Preconceptional health promotion: opportunities abound. Matern Child Health J 2002;6: 71-3.

17. Moos MK. Preconceptional wellness as a routine objective for women's health care: an integrative strategy. J Obstet Gynecol Neonatal Nurs 2003;32:550-6.

18. American College of Obstetricians and Gynecologists. Access to women's health care. ACOG Statement of Policy. Washington DC: American College of Obstetricians and Gynecologists; 2003.

19. Misra DP, Guyer B, Allston A. Integrated perinatal health framework. A multiple determinants model with a life span approach. Am J Prev Med 2003;25: 65-75.
20. Moos MK. Preconceptional health promotion: progress in changing a prevention paradigm. J Perinat Neonatal Nurs 2004;18:2-13.

21. Atrash H, Johnson K, Adams M, Cordero J, Howse J. Preconception care for improving perinatal outcomes: the time to act. Matern Child Health J 2006; 10:3-11.

22. Frey K, Files J. Preconception health care: what women know and believe. Matern Child Health J 2006; $10: 73-7$.

23. Martin JC, Avant RF, Bowman MA, et al; Future of Family Medicine Project Leadership Committee. The future of family medicine: a collaborative project of the family medicine community. Ann Fam Med 2004;2:S3-32. 